\title{
Territorios prehispánicos en las regiones interfluviales, norte de la Amazonía del Ecuador
}

Territoires préhispaniques des régions interfluves, nord de l'Amazonie de l'Équateur

Prehispanic territories from the northern interfluvial regions of the Ecuadorian Amazon

\section{A. Jorge Arellano}

\section{CpenEdition}

\section{Journals}

Edición electrónica

URL: http://journals.openedition.org/bifea/4377

DOI: $10.4000 /$ bifea. 4377

ISSN: 2076-5827

\section{Editor}

Institut Français d'Études Andines

\section{Edición impresa}

Fecha de publicación: 1 mayo 2014

Paginación: 111-132

ISSN: 0303-7495

\section{Referencia electrónica}

A. Jorge Arellano, «Territorios prehispánicos en las regiones interfluviales, norte de la Amazonía del Ecuador », Bulletin de l'Institut français d'études andines [En línea], 43 (1) | 2014, Publicado el 08 mayo 2014, consultado el 05 noviembre 2020. URL : http://journals.openedition.org/bifea/4377 ; DOI : https://doi.org/10.4000/bifea.4377

\section{(c) $(1) \odot$}

Les contenus du Bulletin de l'Institut français d'études andines sont mis à disposition selon les termes de la licence Creative Commons Attribution - Pas d'Utilisation Commerciale - Pas de Modification 4.0 International. 


\title{
Territorios prehispánicos en las regiones interfluviales, norte de la Amazonía del Ecuador
}

\author{
A. Jorge Arellano*
}

\section{Resumen}

Las nuevas evidencias arqueológicas provenientes de las regiones interfluviales del norte de la Amazonía ecuatoriana sugieren una secuencia de ocupación por tradiciones culturales desde el periodo de Desarrollos Regionales. Solo dos sitios tienen relación con el periodo Formativo, el primero, Pata-1, ubicado cerca del piedemonte andino y el segundo, Tiphisca, en la subcuenca del río San Miguel.

La reconstrucción de la secuencia por medio de análisis de material cerámico y fechas radiocarbónicas sugiere que la tradición de cerámica puntuada ocupó indistintamente y de manera aislada áreas interfluviales. Luego la tradición de cerámica polícroma se concentró en las terrazas del Napo y finalmente la tradición de cerámica corrugada-falsocorrugada tuvo control de las regiones interfluviales.

Palabras clave: Ecuador, Amazonía Norte, regiones interfluviales, territorios prehispánicos, tradiciones cerámicas

\section{Territoires préhispaniques des régions interfluves, nord de l'Amazonie de l'Équateur}

\section{Résumé}

Les nouvelles évidences archéologiques trouvées dans les régions interfluviales du nord de l'Amazonie équatorienne suggèrent une séquence d'occupation caractérisée par les traditions céramiques de 
la période de dévoloppement régional. Seuls deux sites sont liés à la période formative, le premier, Pata-1, situé à proximité des contreforts des Andes et le second, Tiphisca, dans le bassin inférieur du fleuve San Miguel.

La reconstruction de la séquence par l'analyse de céramique et la datation par carbone 14 indiquent que la tradition de céramique ponctuée a occupé indistinctement et de façon isolée des zones interfluves. Par la suite, la tradition polychrome s'est concentrée sur les terrasses du Napo et enfin la tradition corrugada-falsocorrugada a pris le contrôle des régions interfluves.

Mots-clés : Équateur, nord de l'Amazonie, régions interfluves, territoires préhispaniques, traditions céramiques

\title{
Prehispanic territories from the northern interfluvial regions of the Ecuadorian Amazon
}

\begin{abstract}
New archaeological evidence from the northern interfluvial regions of the Ecuadorian Amazon suggests a sequence of occupation by different ceramic traditions starting in the Regional Development Period. Only two sites are related to the Formative Period, the first, Pata-1, located near the Andean foothills and the second, Tiphisca, in the San Miguel River sub-basin. The reconstruction of the sequence through the analysis of radiocarbon measurements and ceramic material suggests that the punctuate ceramic tradition was isolated. The polychrome ceramic tradition was mainly along the Napo River, while the corrugated-falsocorrugated ceramic tradition dominated the interfluvial regions.
\end{abstract}

Key words: Ecuador, Northern Amazon, interfluvial regions, pre-Hispanic territories, ceramic traditions

\section{INTRODUCCIÓN}

Las regiones interfluviales en la Amazonía norte del Ecuador se encuentran limitadas por el curso de tres importantes ríos de la cuenca hidrográfica del río Amazonas: Putumayo, Aguarico y Napo. Dos regiones interfluviales mayores al norte del río Napo y cuatro subregiones interfluviales menores al sur, entre el Napo y el río Curaray (fig. 1) fueron en época prehispánica ocupadas por grupos culturales asociados a tradiciones alfareras amazónicas.

En el presente trabajo se definen los territorios de las tradiciones alfareras polícroma y corrugada con su variante falsocorrugada. El corrugado simple es común a lo largo del piedemonte andino y el falsocorrugado es frecuente en las tierras interfluviales al norte del Napo. Esta última técnica emplea tiras de pasta adosadas por presión a las paredes de la vasija facilitando la creación de diseños decorativos.

Por otra parte, se sugiere las relaciones con sitios vecinos en la Amazonía colombiana y tentativamente con el subandino y sur amazónico del Ecuador. Lamentablemente no existen investigaciones arqueológicas en áreas limítrofes del lado brasileño para sugerir relaciones con la Amazonía central. 


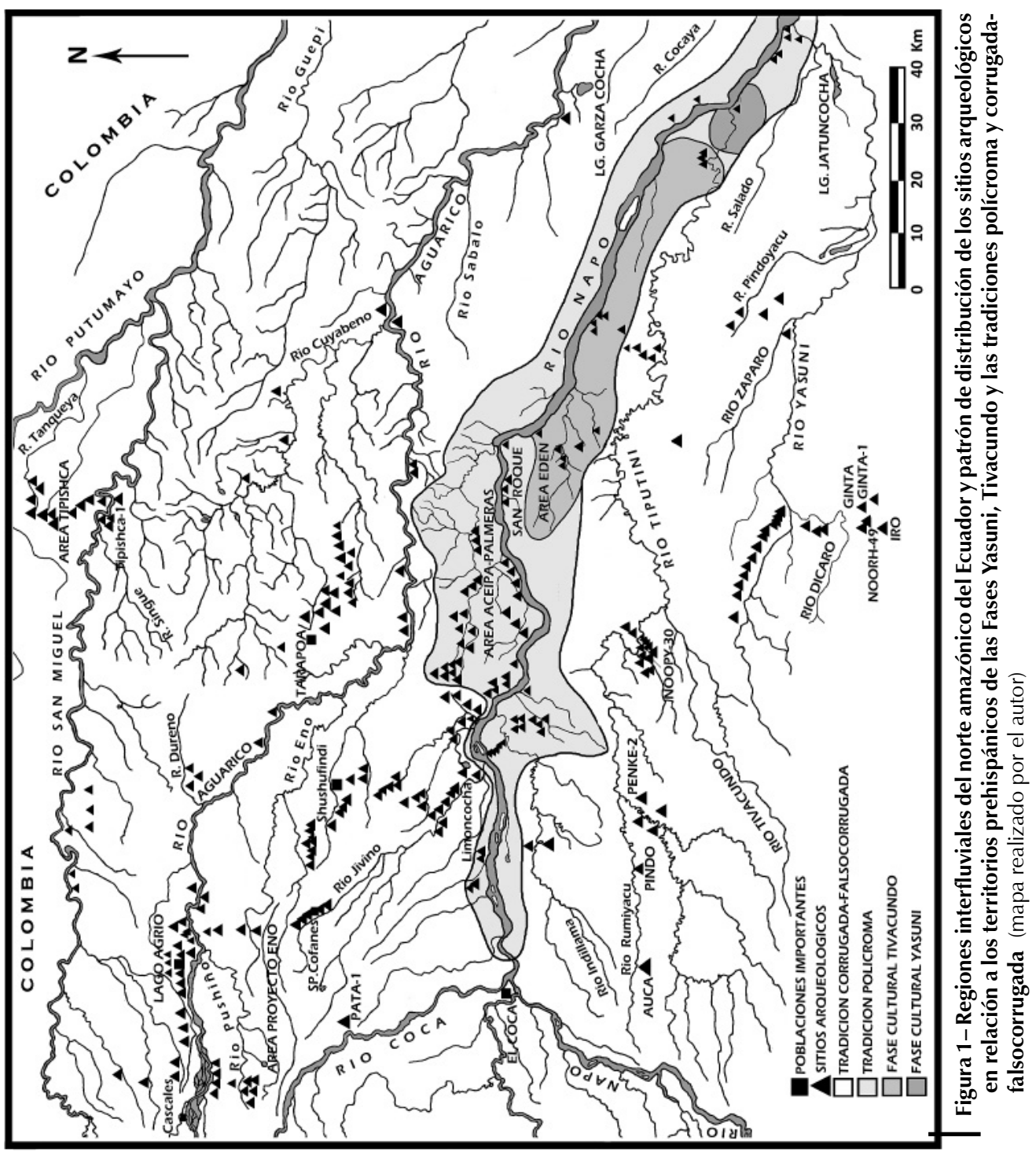


Las evidencias que sirven de referencia para este trabajo, provienen de excavaciones arqueológicas en áreas asociadas a las cuencas del Aguarico y Napo, y de los sitios registrados en estudios de impacto ambiental, desde el río Yasuni por el sur hasta el río San Miguel por el norte (Arellano, 2003a; 2003b; Salazar et al., 1999; 2000; Solórzano, 2006). Es importante mencionar que las normas legales de conservación del medio ambiente proporcionaron a la arqueología amazónica ecuatoriana, un acelerado desarrollo en el método para prospección, muestreo, excavaciones en área y análisis del material cultural. El resultado fue la documentación de una apreciable cantidad de material cultural cerámico y lítico: «200 000 fragmentos de cerámica en el proyecto Eno»(Ochoa, 2007), 150000 en el proyecto Yuturi-Lago Agrio (Arellano, 2009), y alrededor de 33500 en el proyecto Yanaquincha (Arellano \& Tamayo, 2004).

\section{DISTRIBUCIÓN DE LAS EVIDENCIAS ARQUEOLÓGICAS}

Las evidencias arqueológicas de las regiones interfluviales muestran que existieron diferentes patrones de ocupación con relación al medio geográfico y corrientes fluviales (fig. 1). En adición a los patrones de ocupación en ribera y lomas definidos por Netherly (1997), Solórzano (2006: 43; 2007: 123) propone un tercer patrón de carácter lagunar. En consecuencia los patrones de ocupación se clasifican:

- En terrazas altas a lo largo de los cursos de los principales ríos;

- En colinas cercanas a las orillas del río Aguarico;

- En colinas al interior de la ribera derecha del Napo;

- En colinas al interior de las regiones interfluviales y sin acceso directo al río principal como el sitio Tank Farm (Arellano, 2003c);

- En las cabeceras de ríos tributarios;

- A lo largo del curso de ríos tributarios;

- Alrededor de lagunas y pantanos;

- En llanura aluvial interior subsidaria, similar al área de los sitios Aceipa (Arellano, 2009);

- En lomas asociadas a pequeños esteros.

La ubicación geográfica de los sitios está obviamente asociada a la dinámica de interacción con eventos medioambientales.

\section{1. Entre el San Miguel y Aguarico}

Esta región interfluvial se encuentra asociada al río Putumayo a través de la subcuenca del río San Miguel. Las evidencias arqueológicas están agrupadas en tres áreas: la primera en un corredor desde Tipishca hacia el Putumayo; la segunda en el área circundante a la localidad de Tarapoa y la tercera en la orilla izquierda del Aguarico. 
El corredor del Tipishca al Putumayo es importante porque los grupos étnicos del Aguarico se conectaron con la orilla izquierda del Putumayo. Las evidencias de cerámica de la tradición corrugada-falsocorrugada provienen de Singue y están cronológicamente situadas entre 1040 y 1290 d. C. (Aguilera, 2003; Arellano, 2011). La característica de esta cerámica es el acabado de la decoración con estampado de uñas en las tiras alisadas del cuerpo corrugado (Aguilera, 2003: 54). El corrugado simple sobre superficie alisada se encuentra en la mayor parte de las vasijas comunes. El complejo cultural está asociado con urnas funerarias, piezas líticas, un bezote de cuarzo lechoso y una cabeza de delfín modelada en arcilla (Aguilera, 2003: 60). Por su parte el sitio Tipishca 2 fue un taller lítico ocupado entre 930 y 770 a. C. (Aguilera, 2003: 117).

En el área de Tarapoa los asentamientos arqueológicos tienen relación con el complejo de humedales, pantanos y ríos de aguas negras de la subcuenca del río Cuyabeno. Los sitios se ubican en lomas altas de las cabeceras de los ríos (Arellano, 2011: 95). Los contextos de cerámica recuperados entre 20 a $30 \mathrm{~cm}$ por debajo de la superficie, sugieren ocupaciones aisladas. La presencia en diferentes niveles de una sola variedad de vasijas domésticas, indica varias reocupaciones y actividades relacionadas con obtención de recursos de pantanos y humedales. La cerámica pertenece a la tradición corrugada-falsocorrugada. Según Carrera (2003: 202) en las orillas del río Negro se ubica cronológicamente entre 880 y $970 \mathrm{~d}$. C.

En el área de Lago Agrio, orilla izquierda del Aguarico, se determinó una secuencia de reocupaciones por tres fases culturales; dos estuvieron cohabitando y compartiendo recursos. Las fechas radiocarbónicas señalan que esta secuencia se desarrolló entre 1200 y 1400 d. C. La primera fase está representada por una cerámica alisada con decoración punteada y ungular. La segunda por un complejo cerámico con tipos corrugados y falsocorrugados, y la tercera por un complejo de cerámica bicolor y cerámica pulida con engobe de color rojo oscuro (Carrera, 2003; Arellano, 2003c; 2009; 2011). Este último complejo está integrado a nuevos estilos de la tradición corrugada-falsocorrugada que adoptó el uso de pintura roja y el diseño geométrico en zonas. Las diferentes técnicas decorativas en las vasijas no tienen ninguna relación con la fase Pastaza (Carrera, 2003: 191).

Los sitios en la orilla izquierda del Aguarico, desde la ciudad de Lago Agrio hasta la localidad de Cascales, tienen evidencias en capas culturales situadas entre 20 y $30 \mathrm{~cm}$ por debajo de la superficie. En cada uno se presenta el corrugado simple y un falsocorrugado rústico con impresiones ungulares y de dedos. También es común la presencia de cerámica alisada con decoración punteada. Los complejos ocasionalmente tienen fragmentos de «asa puente con pico» o «pico de botella» (Salazar et al., 1999; Carrera, 2003), que sugieren interacción con culturas del subandino que tuvieron contacto con la sierra central. Los sitios multiétnicos son frecuentes en áreas asociadas a pequeñas lagunas, alrededor de las cuales se ubican las evidencias. 


\section{2. Entre el Aguarico y el Napo}

Las áreas importantes en esta región son: las cabeceras del río Pushiño, afluente del Aguarico; el área central asociada a las poblaciones de Eno, Conambo, San Pedro de los Cofanes y Shushufindi; el área asociada a las cabeceras del Itaya y río Niutshindi; el área entre la laguna Limoncocha, el río Jivino y Yanaquincha; a lo largo del río Pañayacu.

Los asentamientos de las áreas central y del río Pañayacu fueron descritos en su oportunidad (Arellano, 2009). El área central está directamente relacionada con la tradición corrugada-falsocorrugada con cuatro fases culturales que representan las reocupaciones de grupos itinerantes de esta tradición. La fase Secoya, la fase Jivino, la fase Pozo Seco y la fase Palora son contemporáneas y situadas cronológicamente entre 1390 y 1440 d. C. Los tipos comunes de cerámica son: alisada, pulida engobada, pintada negro sobre marrón, corrugada y falsocorrugada.

En el área del río Pañayacu se presenta la tradición polícroma con las fases Aceipa y Palmeras (980 a 1180 d. C.), representadas por cerámica pintada con diseños de espirales, círculos, círculos concéntricos y líneas quebradas, en el borde y cuerpo de las vasijas. Las formas de vasijas diagnósticas tienen bordes con labios aserrados o muescas (Arellano, 2009: 121).

Los nuevos datos provienen de tres áreas: San Antonio, San Francisco y San Jacinto (fig. 2), integrados al río Jivino y cabeceras de los ríos Quillapacuy, Ajual y Quinchayacu (Arellano \& Tamayo, 2004). Los sitios San Antonio en la orilla derecha del río Jivino son representativos de una aldea extendida con dos

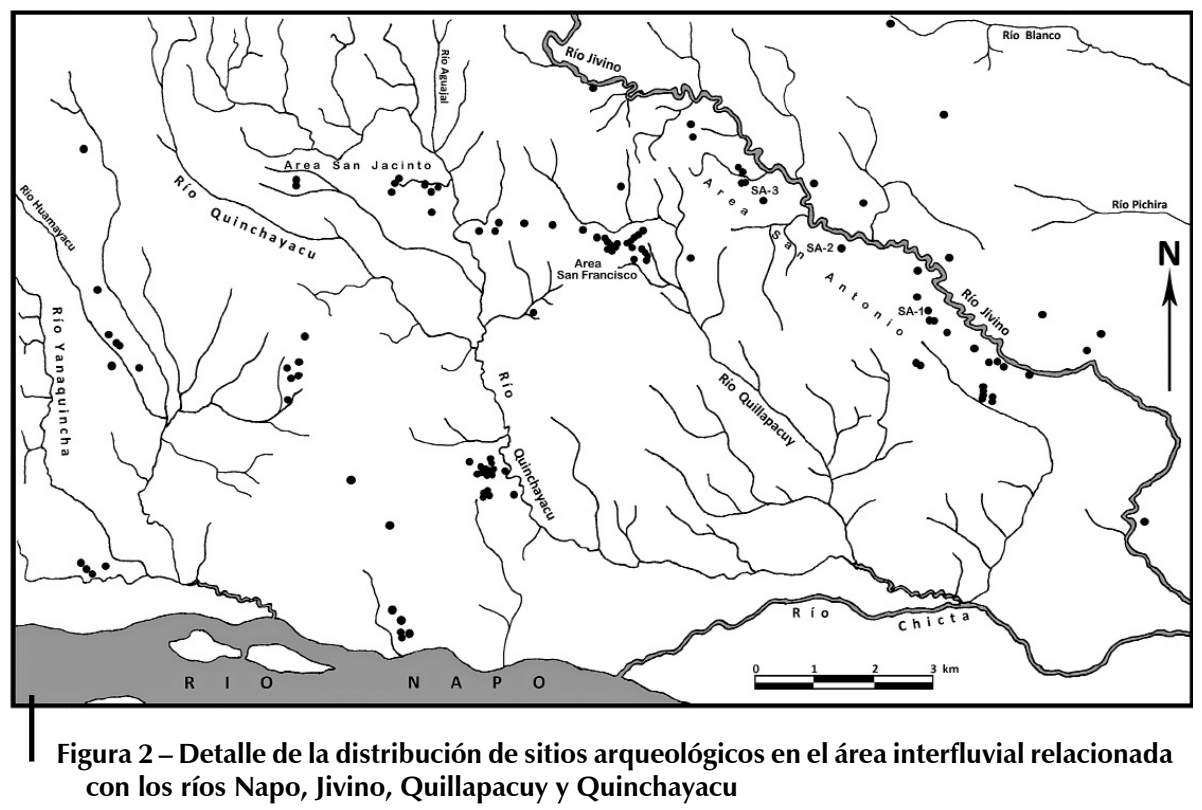

Mapa realizado por el autor 
fases culturales contemporáneas. Las reocupaciones en los sitios indican que practicaron movimientos periódicos, en un modelo similar a lo propuesto para las aldeas en la región de Tocantins en la Amazonía brasileña (Meggers, 1999: 189). Mientras los sitios en las cabeceras del río Quillapacuy tienen características de aldea concentrada con ocupación extendida hacia el río Aguajal. Por su parte los sitios concentrados en el curso medio del río Quinchayacu pertenecerían a una aldea estable sin reocupaciones.

Los trabajos de excavación en los sitios San Antonio, SA-1, SA-2 y SA-3 presentan las siguientes perspectivas: el sitio SA-1 tiene unidades habitacionales con evidencias de reocupación. En dos unidades se determinó una fase cultural con cerámica ordinaria sin decoración, cerámica pulida y engobada en rojo oscuro. Este grupo no tenía ninguna relación con grupos del corrugado; sus evidencias importantes son fragmentos de pico de botella y una variedad de hachas líticas. Posteriormente se presentan compartiendo el área, dos unidades por un grupo caracterizado por cerámica decorada con corrugado simple, falsocorrugado con incisiones oblicuas en diseño reticulado y falsocorrugado con diseño trenzado. Una unidad habitacional tiene evidencias de cerámica polícroma en los niveles inferiores, luego de un nivel estéril se encuentra la tradición corrugada.

En el sitio SA-2 existieron unidades habitacionales donde estuvieron establecidas, primero la tradición polícroma y luego la tradición corrugada-falsocorrugada. Esta secuencia es diferente en SA-3; en dos unidades habitacionales la primera ocupación pertenece a la tradición corrugada-falsocorrugada, posteriormente a su abandono se establece la tradición bicolor. En una tercera unidad es solo bicolor y en la cuarta unidad la primera ocupación es polícroma y sobre esta aparece el corrugado. Esta variabilidad en las secuencias de ocupación es típica del movimiento de aldeas. Es probable que en SA-3 la tradición polícroma que tiene unidades habitacionales separadas, haya sobrevivido hasta el contacto con el corrugado y en cierta manera coexistido. Los fragmentos polícromos diagnósticos son similares a los de la fase Aceipa-Palmeras. Las vasijas tienen aditamentos plásticos y modelados representando la fauna de la región, y diseños geométricos alrededor del cuerpo. En el conjunto doméstico existen cuencos de paredes ligeramente expandidas y pequeñas vasijas globulares con cuello alto.

Los sitios San Francisco en las cabeceras del Quillapacuy están dominados por la tradición corrugada-falsocorrugada. La decoración es sofisticada, sin llegar a la variedad de diseños que se observa en el área de Conambo. Se tiene corrugado simple, corrugado con impresiones dactilares, falsocorrugado con incisiones oblicuas o con puntuaciones y trenzado.

Los sitios San Jacinto son parte de la fase cultural de San Francisco, pero estuvieron compartiendo el área con la tradición polícroma. Los materiales de ambos grupos se encuentran en los mismos niveles culturales.

En las tres áreas, la decoración de la tradición corrugada-falsocorrugada tiene un patrón definido pero diferentes estilos. Las formas de vasijas comunes son: ollas semiglobulares y carenadas con decoración por debajo de los bordes evertidos; vasijas semiglobulares con cuello decorado y bordes evertidos; cuencos 
con corrugado simple de paredes ligeramente expandidas (fig. 3). Las fechas radiocarbónicas que pueden asociarse con estas áreas son las que corresponden a la fase Secoya que tiene un rango cronológico entre 1390 y 1440 d. C. (Arellano, 2009).

Por su parte la concentración de sitios en la orilla izquierda del Napo fueron identificados como parte de la fase Napo. Los sitios relacionados con el río Huamayacu tienen evidencias de la tradición polícroma (Ortiz de Villalba, 1981; Cabodevilla, 1998).
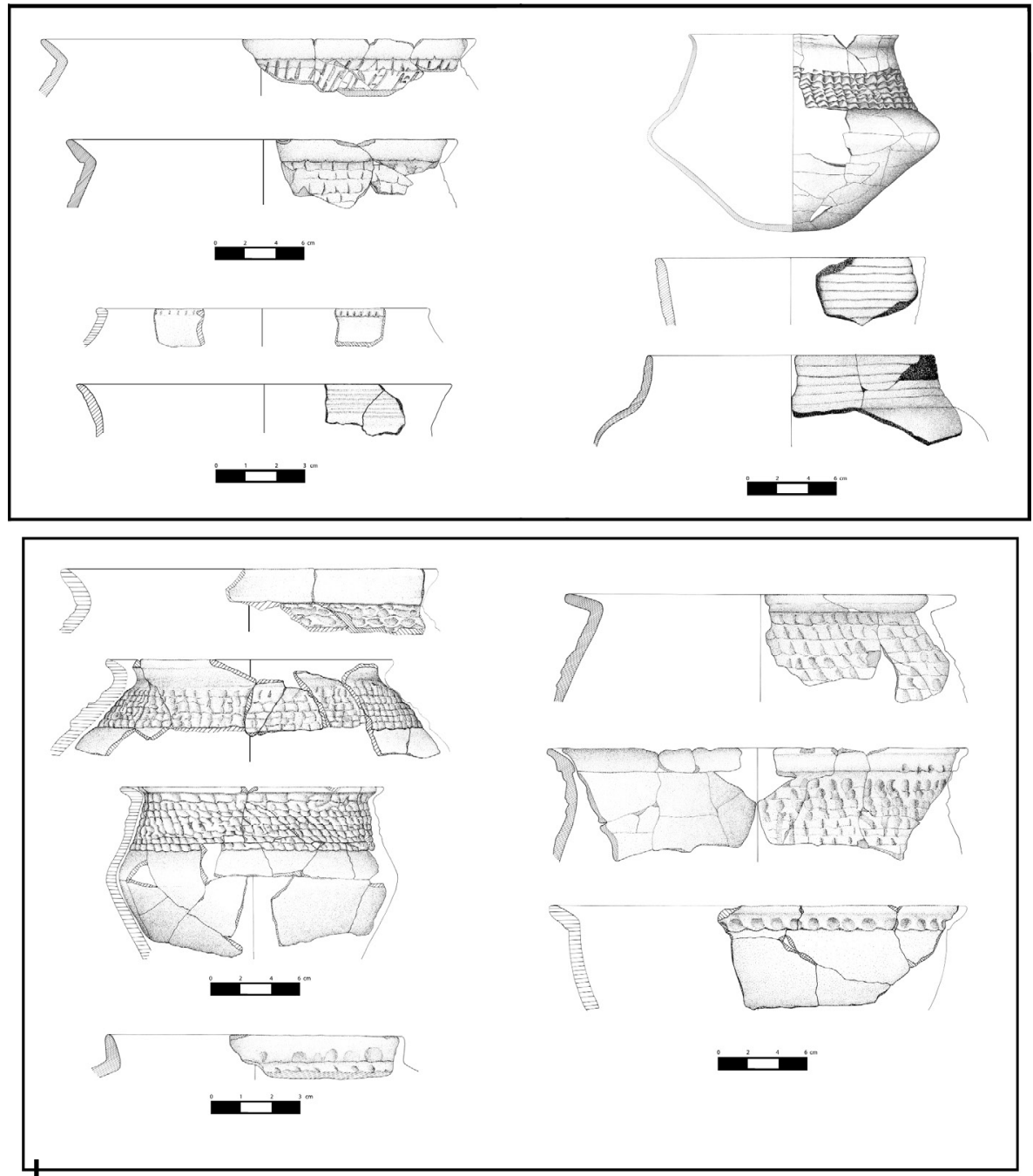

Figura 3 - Región interfluvial Aguarico Napo. Sitios San Antonio. Formas de vasijas con decoración corrugada, falsocorrugada e impresiones dactilares

Modificado de Arellano \& Tamayo, 2004 
Entre «el Río Eno por el Norte, la vía Eno-Lago Agrio al Este, el Río Coca al Oeste y la Reserva Guataraco al Sur» existen otros sitios estudiados por Ochoa (2007: 465) que tienen relación con la tradición corrugada-falsocorrugada. Las evidencias son idénticas en todos los sitios; vasijas carenadas con decoración falsocorrugada en varios estilos por debajo del borde evertido. Sin embargo, en estos sitios aparece una tradición que consideramos anterior al falsocorrugado y se caracteriza por el punteado e inciso-punteado con variaciones combinadas en corrugado simple que, en los sitios Napo descritos por Evans \& Meggers (1968) fue considerada producto de intercambio. En base a fechas radiocarbónicas, Ochoa (2007: 478479) presenta una secuencia de cuatro períodos: el primero entre 250 y 640 d. C. se encuentra en los sitios Guayabo y Nogales, con presencia de «corrugado, falsocorrugado, exciso, inciso e impresiones ungulares». Un segundo período, que va de 520 a 780 d. C. se presenta en Itayacu, «aparecen el inciso punteado, corrugado con inciso, falsocorrugado con inciso, pintura roja y negra». El tercer período va de 910 a 1410 d. C. en los sitios Llurimagua, Itayacu, Chiliuza, Pozo Rojo, Jivino Rojo 2 y Conambo. Estos sitios presentan 17 decoraciones distintas y cerámica intrusa negro sobre rojo estilo Aguarico. Finalmente el cuarto período va de 1420 a 1650 d. C. en el sitio Plataforma Pata 3, donde «predominan corrugados, impresiones dactilares y ungulares, inciso punteado, inciso puntuado con corrugado». Además existen «cuencos y platos con decoraciones corrugadas al exterior y negativas al interior, pintura irisdicente o pintura roja».

De las fechas radiocarbónicas mencionadas por Ochoa (2007: 478), y aunque no se conoce si las decoraciones excisa, incisa e ungular coexisten con el corrugadofalsocorrugado, se infiere dos ocupaciones diferentes en su primer período, una temprana y otra tardía. Mientras en el tercer período, parecen existir tres reocupaciones en el área. También otro grupo estuvo habitando en el área y está representado por cerámica decorada en negro sobre rojo.

\section{3. Sur del río Napo}

Las investigaciones arqueológicas en la ribera derecha del Napo se iniciaron con los trabajos de Evans \& Meggers en 1968 en los sitios localizados en el área de Nuevo Rocafuerte. La secuencia de cuatro fases culturales determinada en esta área, Yasuni (40 a. C.-140 d. C.), Tivacundo (480-620 d. C.), Napo (11681480 d. C.) y Cotacocha, es el marco de referencia del desarrollo cultural en la Amazonía ecuatoriana.

En las subregiones interfluviales al sur del Napo los sitios arqueológicos, en general están situados en terrenos altos asociados a pequeñas Ilanuras aluviales de inundación. Netherly (1997: 45) sugiere para el área ribereña del Napo jerarquías de sitios «con algunos centrales y otros periféricos de menor jerarquía y que las áreas vacías servirían como cotos de caza»; también menciona que los sitios tienen varias reocupaciones. Los sitios en el área ribereña pertenecen a la fase Napo, pero contienen «cerámica más ruda» y también presentan evidencias de devitage de obsidiana (Netherly, 1997: 48). 
En el paisaje de lomas ribereñas de la subcuenca del río Indillama, afluente del Napo, los materiales arqueológicos aunque no permiten inferir su filiación cultural muestran tres «momentos de ocupación», la temprana que tiene 30 a $60 \mathrm{~cm}$ de profundidad, la segunda intermedia entre 15 y $28 \mathrm{~cm}$ y la tardía entre 0 a $14 \mathrm{~cm}$ (Netherly, 1997a: 15).

Entre los nuevos estudios arqueológicos en la orilla derecha del Napo se encuentran el efectuado en San Roque (Arellano, 2009) y el realizado en el asentamiento arqueológico de Eden (Salazar \& Ochoa, 2003). Las evidencias culturales de Eden provienen de seis sitios: Eden, Yuturi I, EPF 1, EPF 2, Yuturi II y Canoayacu (Ochoa, 2003). El análisis de los restos culturales determinó que con excepción de Yuturi I que pertenece a la fase Napo, los demás pertenecen a la fase Tivacundo (Salazar \& Ochoa, 2003). Por otra parte, los sitios localizados en la comunidad San Roque pertenecen a la fase San Roque (570 a 880 d. C.), situada cronológicamente entre las fases Tivacundo y Napo. Los cortes estratigráficos de las excavaciones en San Roque muestran la siguiente secuencia: la fase Napo se encuentra en los niveles superiores, en los niveles medios la fase San Roque y los niveles profundos tienen evidencia de una cerámica decorada con puntuaciones e incisiones (Arellano, 2009).

Al sur de las terrazas del Napo las evidencias son escasas y no existe un estudio metódico de los restos culturales. Los sitios se encuentran en la parte superior de colinas y lomas cercanas a llanuras de inundación de los ríos. En el área Penke situada en la orilla derecha del Tiputini, el sitio Penke-2 proporcionó material cerámico y lítico que fueron asociados con la fase Tivacundo por la presencia de un fragmento de colador (Villalba, 2000: 12). No obstante vasijas «coladores» se encuentran en todas las fases de la tradición polícroma.

En el área Pindo, entre el Tiputini y su tributario el Rumiyacu, fueron registrados siete sitios con evidencias pero sin filiación cultural (Sanchez, 2002). Los fragmentos fueron considerados producto de arrastre fluvial por encontrarse en una área no apta para ocupación (Dominguez, 2001a). En Pindo-Il se detectó material cultural que sugiere actividades domésticas de una sola ocupación (Dominguez, 2001b). En las cabeceras del río Rumiyacu el área denominada Auca contiene limitadas evidencias culturales, «12 fragmentos de cerámica no diagnóstica», que no permiten sugerir la fase cultural al que pertenecen (Villalba, 2003).

La mayor concentración de sitios se encuentra en la confluencia del río Bogi con el Tiputini. El informe de análisis de la cerámica del sitio NOOPY-30 menciona que existe una apreciable cantidad de fragmentos que no definen una fase cultural (Guamán \& Netherly, 1996). Por su parte en dirección al Tivacuno «la prospección reveló que había por lo menos tres ocupaciones» con la «más reciente de hace unos 20 años» (Netherly, 1997: 49). En el área Dícaro los sitios contienen material cerámico de pequeño tamaño y no permiten inferir su correspondencia a una fase en particular.

En la información de los sitios que se ubican en las colinas a lo largo del Yasuni se mencionan la presencia de saladeros y de un taller lítico (Netherly, 1997) y en el área del río Dícaro «los restos cerámicos sugieren filiaciones con el Sur y además constituye la frontera huarani-záparo» (Netherly, 1997: 49). 
Al sur del río Dícaro, en el área Ginta se tiene por vez primera descripción de las características de una cerámica que proviene de los interfluvios al sur del Tiputini. La cerámica del sitio Ginta-1 presenta una decoración corrugada que fue clasificada como «de uso domestic» (Echeverria, 2001: 10). Mientras en el sitio NOORH-49 se recuperó un sello inciso, los restos de cerámica son del estilo «Napo», y se obtuvo una fecha radiocarbónica de $950 \pm 70$ d. C. (Netherly, 1997a: 50).

Hacia la boca de Tiputini (fig. 4), al margen de los sitios descritos por Evans \& Meggers (1968) fueron registrados tres sitios al norte del Tiputini asociados a pequeños tributarios del Napo; seis sitios en el curso del Tiputini y cinco en el curso del Yasuni. El sitio NKR-8 ubicado en una colina proporcionó evidencias de una ocupación aislada. El material cultural compuesto por fragmentos de cerámica y una pieza lítica fue recuperado a $20 \mathrm{~cm}$ de la superficie mediante una cuadrícula de excavación. Las formas reconstruidas corresponden a vasijas globulares de acabado alisado, con cuellos decorados con impresiones ungulares y bordes evertidos. La pieza lítica es una preforma de una pequeña hacha de cuello constreñido, pero el dato importante se refiere al tipo de roca, calcedonia, muy rara en esta parte de la Amazonía (Arellano, 2003a). Otro sitio que podría tener conección con el anterior, es el NKR-7 ubicado en una terraza de $208 \mathrm{~m}$ de altura en un afluente de la orilla derecha del Tiputini. En este sitio, a 15 cm

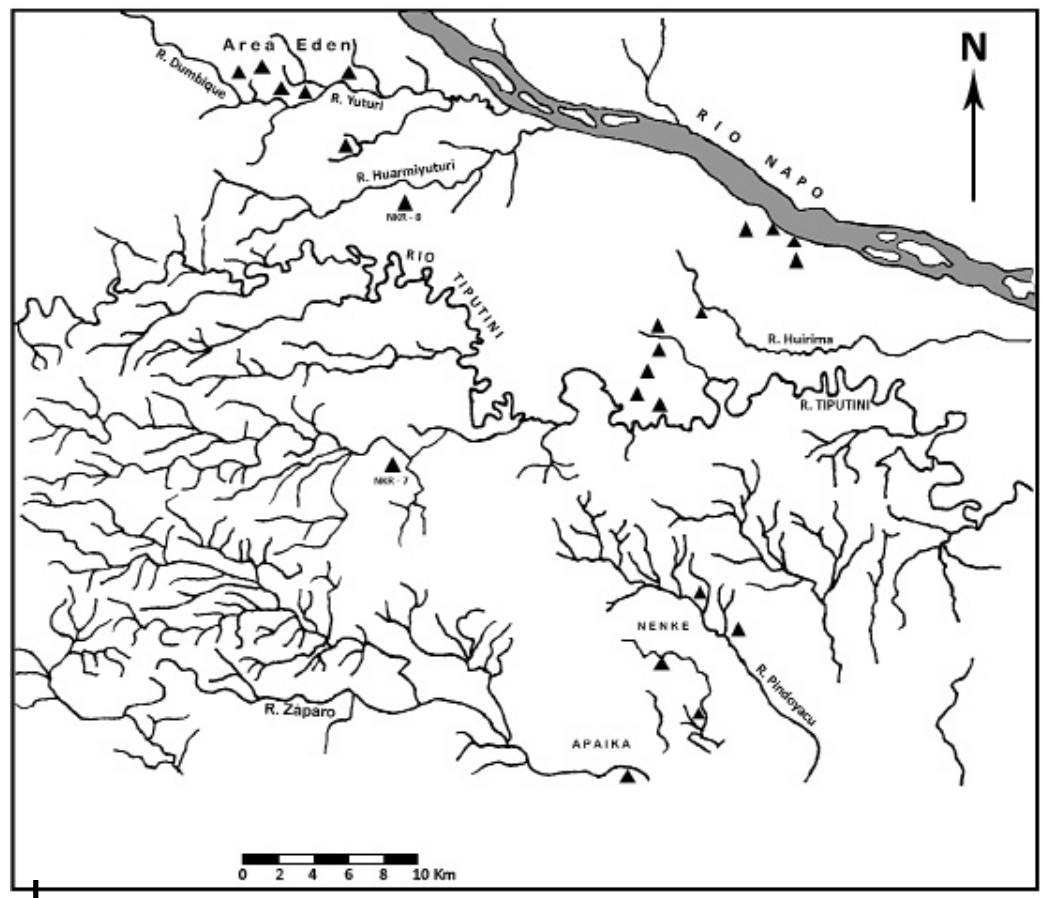

Figura 4 - Detalle de la distribución de sitios entre los ríos Yuturi y Tiputini

Mapa realizado por el autor 
de profundidad se recuperaron desechos líticos de cuarzo, cuarzo lechoso, calcedonia y cuarcita. Aunque se trata de «devitage», existen microrraspadores circulares y lascas útiles que tuvieron la probabilidad de ser usados como artefactos cortantes o perforadores (Arellano, 2003a). Los demás sitios de esta área presentan evidencias de fragmentos de cerámica y hachas líticas. La cerámica usualmente no es decorada y tiene un acabado alisado. La característica común entre los sitios es que el material cultural se encuentra en la parte superior de colinas.

En el área Nenke se efectuaron dos estudios de prospección arqueológica. El primero con solo dos pruebas de pala positivas en las que se ubicaron restos de carbón (Delgado, 2002: 12). En el segundo se implementaron 63 pruebas de pala, con 8 resultados positivos. El rescate arqueológico tuvo lugar por medio de tres unidades de excavación. En la primera unidad se ubicaron dos vasijas junto a restos de carbón y fragmentos de huesos quemados (Acuña \& Delgado, 2002: 7). Las vasijas de forma globular tienen cuellos decorados con la técnica del falsocorrugado y bordes evertidos. El análisis palinológico del contenido de una de ellas y una datación radiocarbónica de $1150 \pm 10$ BP (910 a 1290 d. C. calibrado) (Delgado \& Acuña, 2003: 10), proporcionan las mejores variables culturales obtenidas para la región del río Tiputini. En particular, el análisis palinológico tiene relevancia por estar asociado a una vasija de la tradición corrugada-falsocorrugada y por la diversidad de taxas que fueron determinadas. Las que sugieren empleo de especies vegetales para una actividad doméstica y otras que sugieren horticultura o agricultura incipiente son: Phytelephas sp. (Tagua), probablemente empleado por los grupos étnicos como fertilizador del suelo, especialmente en esta área relacionada con llanura de inundación; Astrocaryum sp. es una variedad de palma utilizada por los nukak de Colombia para la elaboración de hamacas; Cyperus sp., otra variedad de palma utilizada para esteras; Celtis schippi, actualmente empleada para postes de las viviendas; Curcubita sp. y Lagenaria sp. ampliamente utilizadas en la Amazonía. Sin embargo por las implicancias en la interpretación socioeconómica la taxa más importante es Zea mays. Este dato, aunque muy aislado, asociado a las evidencias de maíz y guaba (Inga sp.) obtenidas en Huapula en el alto Upano (Rostain, 1999: 244), sugieren que el maíz fue conocido y consumido al sur del Napo.

Finalmente, en los reconocimientos arqueológicos en el área Apaika se registró un sitio con tres piezas líticas (Ochoa, 2000), y en dirección al río Yasuni seis sitios con evidencias de cerámica con engobe leonado (Almeida, 2001).

\section{SECUENCIA DE OCUPACIÓN DE TERRITORIOS PREHISPÁNICOS}

La secuencia de ocupación y territorios de los grupos prehispánicos en las regiones interfluviales tiene relación con la ubicación geográfica de las evidencias de las tradiciones cerámicas (fig. 1). En el cuadro cronológico (fig. 5) se observa que dos sitios pertenecen al período Formativo: Tipishca, un taller lítico al norte del río San Miguel y Pata-1 en la orilla izquierda del río Coca. El sitio Pata-1A estudiado por Echeverría (1999; 2006) y mencionado por Rostoker (2003), es el primer sitio 


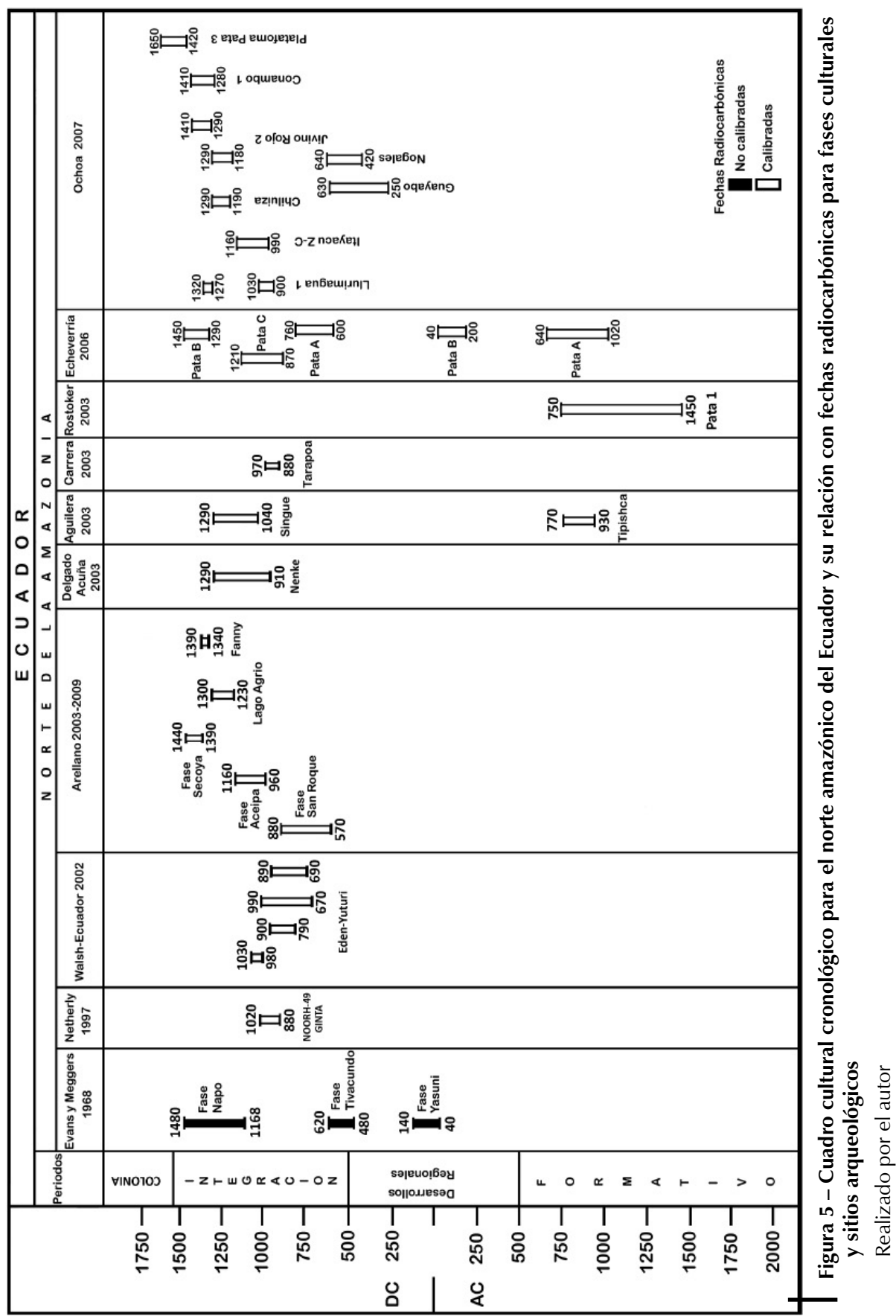


en la secuencia cultural con un complejo formativo. El material está conformado por cerámica de paredes delgadas engobada en rojo pulido, un fragmento de asa pico de botella y una hacha lítica (Echeverría, 2004: 68). En el sitio existen otras ocupaciones luego de un hiato estratigráfico con sedimentos que sugieren una extensa inundación. Luego vuelve a ocuparse entre 600 y 760 d. C. por una fase de la tradición polícroma del estilo Napo conformada por cerámica delgada decorada con bandas blancas y rojas (Echeverría, 2004: 68). Este complejo probablemente pertenece a la fase San Roque. Las otras ocupaciones Pata-1B (1290 a 1450 d. C.) y Pata-1C (870 a 1210 d. C.) pertenecen a una coexistencia de las tradiciones punteada-ungular y corrugada-falsocorrugada (fig. 6). La decoración punteada e incisa en el material de estas dos ocupaciones muestran cierta similitud con la decoración punteada zonal descrita en la fase Pastaza (Porras, 1975: Lámina 1).

El territorio de la primera fase cultural de la secuencia, Yasuni, está restringido a la boca del río Tiputini en el área circundante a los sitios mencionados por Evans \& Meggers (1968: 14). Se caracteriza por una cerámica decorada con diseños geométricos realizada con incisiones y puntuaciones, y una decoración ungular en bordes y carenas de vasijas.

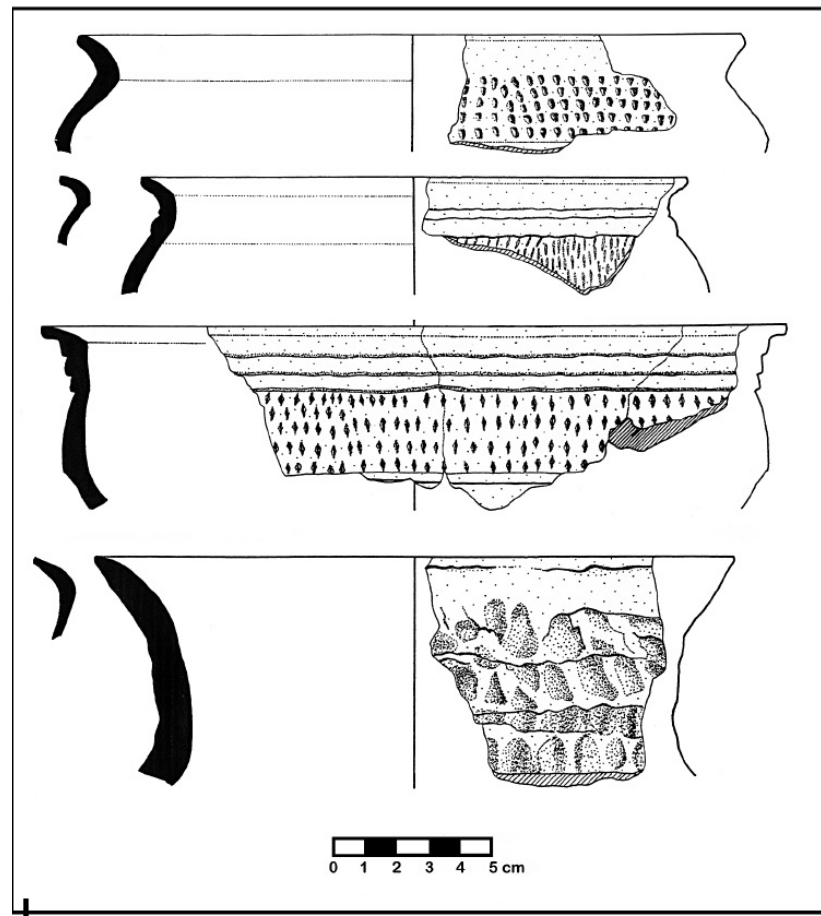

Figura 6 - Región interfluvial Aguarico-Napo, Sitio Pata-1. Formas de vasijas con decoración punteada, ungular y falsocorrugada con impresiones dactilares

Modificado de Echeverría, 1999 
La siguiente fase Tivacundo presenta una cerámica decorada con incisiones en zonas geométricas pintadas en rojo, y cerámica pintada con diseños geométricos en rojo (Evans \& Meggers, 1968: 29). Los sitios tipo se ubican sobre una terraza en la orilla derecha del río Tiputini. A partir de estos sitios el territorio de esta fase fue ampliado hacia el curso del río Yuturi donde se ubican los asentamientos de Eden. Según Ochoa (2007: 481) uno de ellos «constituye el más grande asentamiendo Tivacundo, cuya ocupación fue datada entre el 670-990 AD».

Posteriormente, en la secuencia se tiene al territorio de las fases de la tradición polícroma: San Roque, Napo, Aceipa-Palmeras. Es probable que los sitios circunscritos al área situada entre la boca del Coca y el río Indillama con evidencias del estilo «Napo» se ubiquen cronológicamente antes de la fase San Roque. De manera que el territorio de la tradición polícroma comprende las terrazas de ambas orillas del Napo y eventualmente podría extenderse hacia la orilla izquierda del Coca. Esta apreciación fue en su momento confirmada por Solórzano (2006) en la interpretación de la distribución de asentamientos en la orilla izquierda del Coca y sur del Napo.

Se completa la secuencia territorial con la tradición corrugada-falsocorrugada que tomó el control de las regiones interfluviales y evitó los territorios que se encuentran en las orillas del Napo. Por el momento es complicado determinar las etapas que utilizó para controlar estas regiones. En la región Aguarico-Napo, en el área circundante al río Eno fue posible definir una secuencia cultural entre 1230 d. C. y 1440 d. C. con las fases Secoya, Jivino, Pozo Seco y Palora (Arellano, 2009). También al suroeste, en el área que limita con el río Coca se determinó una secuencia continua de cuatro períodos desde 250 d. C. hasta 1650 d. C. (Ochoa, 2007), el último integramente de la tradición corrugada-falsocorrugada.

\section{DISCUSIÓN Y CONCLUSIONES}

El cuadro cronológico (fig. 5) sugiere la probabilidad de que el curso del río Napo haya sido ocupado desde el Formativo. Por su parte, los picos de botella registrados en Pata-1, SA-1 y orillas del Aguarico (Carrera, 2003) que son cercanamente similares a los de Cotocollao (Porras, 1982; Villalba, 1988), hacen pensar en un contacto temprano con las hoyas interandinas.

Sin embargo, la ocupación en el Napo se hace más evidente alrededor de 40 a. C. hasta 140 d. C. en el período de Desarrollos Regionales; luego existe un hiato y vuelve a ser ocupado en el año 480 d. C. y continúa hasta 1480 d. C. El curso del Aguarico y las tierras interfluviales son ocupadas en el período de Integración apróximadamente desde 900 d. C. hasta 1450 d. C.

En el período de Integración se observa dos diferentes grados de desarrollo cultural. Al norte del Napo las sociedades estuvieron en control de recursos renovables. Los espacios interfluviales fueron estables y no existió una extrema variabilidad medioambiental en los períodos de inundaciones o en su caso por eventos volcánicos. La densidad de evidencias de ocupaciones en un radio geográfico 
restringido a algunas áreas, indica su preferencia para reocupaciones y retorno de movimientos itinerantes. Al mismo tiempo muestra la común necesidad de ubicar las habitaciones sobre terrenos altos, característica también determinada por Solórzano (2007: 117). Al parecer en estos paisajes «alcanzaron un estable y autosustentable asentamiento» y se llegó a conocer la «interacción entre suelo, clima y biota» (Meggers, 1992: 203).

Al sur del Napo, los datos geomorfológicos y medioambientales indican que las inundaciones fueron frecuentes, extensas y sin drenaje. Los ríos con dirección al Marañón son divagantes y erráticos, con abundancia de meandros. Las áreas estables son las terrazas de la orilla sur del Napo, y un corredor de colinas que forman un paisaje ondulado hasta la boca del Tiputini.

Las evidencias arqueológicas dispersas y pertenecientes a complejos culturales tardíos, indican que el medio ambiente de llanura aluvial amazónica, «no fue un ambiente propicio para asentamientos permanentes», porque está conformado por una variedad de microambientes con una exclusiva y particular biodiversidad en constante renovación y cambio. En el análisis estadístico de la distribución de asentamientos Solórzano (2006: 52) realiza «una analogía etnográfica con los Waorani» indicando «que las formas de asentamientos corresponderían a grupos itinerantes, que se mantenían en forma estable durante lapsos determinados para luego proceder a su abandono». Agregando que este modo de vida «es una forma de manejo ecológico, evitando la total desaparición de los recursos bióticos».

El orígen de las migraciones de las tradiciones puntuada-ungular, polícroma y del corrugado-falsocorrugado, de o hacia la Amazonía ecuatoriana tiene una serie de controversias por la variabilidad de los datos. La datación de la tradición puntuadaungular proviene del sitio Pata-1, ubicado en el curso del río Coca (Echeverría, 1999; Rostoker, 2003). A pesar de ser un sitio formativo, 1450 a. C. a 750 a. C. (Rostoker, 2003: 573), es difícil sugerir que fue el foco de la expansión punteadaungular. No obstante es importante mencionar que este tipo de cerámica se presenta invariablemente en los niveles profundos de los sitios ubicados en las orillas del Napo.

La trayectoria de dispersión de la tradición polícroma fue en principio tratada por Meggers \& Evans (1958) sugiriendo que «siguiendo el curso del Napo proviene de los contrafuertes andinos de la región ecuatoriana». Posteriormente, Meggers (1999: 281) con las evidencias del Bajo Amazonas indica que «la tradición polícroma podría haber iniciado su migración o dispersión río arriba», desde «la unión del río Negro con el Amazonas». Actualmente con las nuevas evidencias de sitios Napo ubicados en la boca del río Coca (Echeverría, 2004), es evidente que la tradición polícroma tenía contacto con las culturas de la franja marginal del subandino.

Por su parte, el origen y trayectoria de la tradición corrugada-falsocorrugada tiene relación con las diferentes características tecnológicas y decorativas empleadas en la elaboración de los complejos cerámicos. El apreciable porcentaje de datos arqueológicos del piedemonte amazónico y regiones amazónicas del norte de Perú, Ecuador y sur de Colombia, permiten configurar un panorama con varias 
posibilidades. En principio está prácticamente aceptado que la fase Pastaza no tiene ninguna relación con la tradición corrugada-falsocorrugada (De Saulieu, 2006; Ochoa, 2007) y que el corrugado que se presenta en el sur ecuatoriano es bastante simple, tosco y rústico en su decoración. Los últimos estudios efectuados por De Saulieu (2006) en la colección de la fase Pastaza de Porras (1975), determinaron que la fase Pastaza está asociada al complejo cerámico PastazaKamihun, que en parte tiene excisiones e incisiones geométricas y pintura roja, y que el corrugado de la colección es tardío.

En este sentido, a partir de la propuesta de un Horizonte Corrugado a lo largo del piedemonte subandino (Guffroy, 2006), se puede sugerir varias rutas de dispersión del corrugado, considerando que «los conjuntos cerámicos tienen en cada región caracteres singulares que probablemente reflejan situaciones particulares y diversos procesos culturales de adquisición» (Guffroy, 2006: 80). Al norte del Aguarico es factible considerar una relación con los sitios ubicados en la subcuenca del Putumayo, municipio de Orito, sur de Colombia (Cifuentes, 2007a; 2007b), que tienen evidencias de cerámica corrugada con una cercana similitud a la presente en los sitios de la orilla izquierda del Aguarico. Las primeras fechas radiocarbónicas que tienen relación con esta tradición provienen de la zona denominada trapecio amazónico de Colombia. Los sitios 10 y 11 en la planicie aluvial del río Loretoyacu presentan evidencias de cerámica con desgrasante de cerámica, decoración corrugada simple y falsocorrugada con incisiones diagonales, y dataciones entre $615 \pm 125$ d. C. y $700 \pm 130$ d. C. (Bolian, 1972: 5). En esta misma área, tres nuevos sitios en el paisaje de tierra firme Igarapé Takana asociado a «terras pretas» (Morcote-Ríos \& Sicard, 2012: 154), presentan un panorama diferente porque no existe evidencia de la tradición corrugada sino más bien de una tradición que empleaba el uso de cariapé como desgrasante y engobe de diversas tonalidades en vasijas globulares con soporte anular. Las evidencias de cerámica en una capa cultural dispuesta entre 25 y $35 \mathrm{~cm}$ de profundidad, tienen dataciones en un rango que va de 680 a 890 d. C. (Morcote-Ríos \& Sicard, 2012: 162).

Por consiguiente existe la presencia de dos tradiciones en la zona, la primera asociada a la tradición corrugada y que probablemente abandonó el área, y la segunda a una tradición bicolor. Esta última, por los datos de Igarapé Takana, sitio que se encuentra en el territorio Aparia, podría pertenecer a un grupo derivado de esta etnia. Además, ya estuvo involucrada en el consumo de maíz y no solo de yuca (Morcote, 2006: 257; Morcote-Rios \& Sicard, 2012: 155). El sitio también podría estar asociado a los yagua y luego tikuna (Morcote-Rios \& Sicard, 2012: 48) y desplazamientos muy bien documentados de la etnia Tikuna en el siglo XVIII (Zárate, 1998).

En el área de alto y bajo río Purité, los sitios arqueológicos Maicura (970-1190 d. C.) y Omé que tienen dos etapas de ocupación, 660-775 d. C. y 1265-1405 d. C. (Morcote-Rios, 2008: 81), presentan complejos de cerámica diferentes, sugiriendo cohabitación de dos grupos no afines. La cerámica del sitio Maicura tiene características cercanas a las tradiciones polícromas de las regiones interfluviales del Aguarico y Napo; la diferencia es el empleo del desgrasante en la 
pasta, una mezcla de cariapé, arcilla seca triturada, elementos de palma y cauixí. Mientras en Omé la cerámica no presenta decoración y el desgrasante es cariapé (Morcote-Rios, 2008: 179).

En las subcuencas de los ríos Guamues y Orito en la Amazonía colombiana las variedades de falsocorrugado que tienen impresiones dactilares y otras que tienen bandas corrugadas divididas con incisiones formando cuadriculados (Uribe, 1980-1981; Cifuentes, 2006), son cercanas a los estilos del Aguarico. Estas variedades presentes en el denominado complejo Mocoa tienen cuatro fechas radiocarbónicas: $1020 \pm 50$ d. C.; $1140 \pm 50$ d. C.; $1120 \pm 70$ d. C. y $1529 \pm 70$ d. C. y fueron interpretadas como una secuencia del movimiento de ocupación desde la Amazonía baja hacia el piedemonte subandino (Cifuentes, 2006: 126). Sin embargo se observa en la cerámica de los sitios de la cuenca del Putumayo y subcuenca del San Miguel de la parte colombiana, el carácter rústico en la decoración corrugada y falsocorrugada, en relación a la variedad de estilos y acabado que se presentan en las áreas de: Lago Agrio, Eno, Conambo y Jivino.

Al sur del Napo, según Guffroy (2006: 357) la presencia del Horizonte Corrugado en el suroriente del Ecuador está relacionada a grupos «protohistóricos y modernos del idioma jívaro» y su arribo «a la ceja de montaña entre la Cuenca alta del Río Napo y el Río Marañon» ocurriría después del siglo XVII d. C. Además que otros movimientos de grupos de la «subfamília lingüística jibaroan hacia el Norte del Ecuador y Colombia pudo haber tenido lugar después del siglo X». Si bien está claro que existen varios grupos al interior de la tradición corrugadafalsocorrugada, las evidencias no son definitivas para integrar toda la tradición a una sola familia lingüística. Especialmente porque la variedad de diseños decorativos del falsocorrugado de las regiones interfluviales al norte del Napo, no tienen ninguna similitud con el corrugado del subandino y sur del Napo. Por otra parte, tradicionalmente los pueblos jívaros son inestables y sus asentamientos son estacionales. El efectivo comportamiento para migraciones y estabilidad en territorios desconocidos está asociado a grupos que buscan como recompensa algún recurso conocido pero desaparecido en una área; se debe añadir la búsqueda de mujeres fuera del clan familiar, de manera similar a lo registrado en los grupos taromenae donde existen mujeres de origen teageri, haciendo que los clanes sean mezclados (Coelloni \& Proaño, 2010).

En el lejano sur, en el subandino amazónico, el sitio Huapula que forma parte del complejo de montículos del Sangay (Porras, 1987; Salazar, 1999), el corrugado vendría a ser parte de la última ocupación en la «cima del montículo» entre el «692-892 d. C.» y tendría características similares a las ocupaciones jívaras (Rostain, 1999: 247; 2006: 340). Asociada se presenta cerámica con «pintura roja y blanca» y con «pintura negra y roja» (Rostain, 2010: 678).

En conclusión, las regiones interfluviales del norte amazónico fueron ocupadas por una secuencia de tres tradiciones culturales, subdivididas en fases culturales que evolucionaron hacia grupos étnicos compactos en territorios definidos, mientras al sur del Yasuni fueron tribales. En el norte tuvieron como característica socioeconómica el manejo periódico de recursos naturales por 
medio de movimientos migratorios de reocupación, en un territorio delimitado y expandido por la capacidad interna de núcleos familiares y parentesco. Esta última particularidad puede observarse en la repetición y creación de diseños decorativos en el falsocorrugado.

En el sur fueron pequeños grupos tardíos e itinerantes, asociados a las migraciones étnicas del siglo XVI (Renard-Casevitz et al., 1988) desde la zona amazónica peruana y luego a la aparición en las estribaciones subandinas de la denominada cultura jívara. Al norte del Aguarico las migraciones tardías parecen provenir de la Amazonía colombiana.

\section{Agradecimientos}

Deseo expresar mis agradecimientos al Departamento de Antropología del Museo Nacional de Historia Natural de la Smithsonian Institution, en particular a la Dra. Mary Jo Arnoldi, Chair del Departamento, al Dr. Dennis Stanford, Dr. Ronald Bishop y Dr. Paul Taylor, por darme la oportunidad de ser parte de la comunidad de investigadores de esta prestigiosa Institución. De Igual manera en Ecuador, quiero agradecer al Dr. Mark Turber, Ing. Peter Ayarza y Lic. Cyana Zambrano, de Walsh Environmental Scientists and Engineers. Mis agradecimientos a los revisores por sus valiosas sugerencias, ideas y comentarios, para mejorar este artículo.

\section{Referencias citadas}

ACUÑA, F. \& DELGADO, F., 2002 - Informe de monitoreo arqueológico en la plataforma Nenke, en el Bloque 31 de la Provincia Francisco de Orellana; Quito: Instituto Nacional de Patrimonio Cultural. Daimi Service S.A.

AGUILERA, M., 2003 - Del San Miguel hacia el Norte. In: Cuyabeno Ancestral (M. Aguilera, J. Arellano, J. Carrera \& R. Jones, eds.): 49-122; Quito: Ediciones Simbioe, GAIA, WALSH Ecuador.

ALMEIDA, E., 2001 - Reconocimiento y rescate arqueológico en Apaika Norte y Sur, Bloque 31; Quito: Instituto Nacional de Patrimonio Cultural. Daimi Service S.A.

ARELLANO, A. J., 2003a - Diagnóstico y reconocimiento arqueológico preliminar de las alternativas para la construcción del campamento base, sus vías de acceso y alternativas del oleoducto de evacuación para la infraestructura del plan de desarrollo del Bloque 31; Quito: Informe al Instituto de Patrimonio Cultural.

ARELLANO, A. J., 2003b - Prospección Arqueológica en la Plataforma Eden-G del Bloque 15 y Reconocimiento Arqueológico en su Vía de acceso, Provincia Francisco de Orellana; Quito: Informe al Instituto Nacional de Patrimonio Cultural.

ARELLANO, A. J., 2003c - Lago Agrio. In: Cuyabeno Ancestral (M. Aguilera, J. Arellano, J. Carrera \& R. Jones, eds.): 123-164; Quito: Ediciones Simbioe, GAIA, WALSH Ecuador.

ARELLANO, A. J., 2009 - Culturas Prehispánicas del Napo y Aguarico, Amazonía Ecuatoriana, 150 pp.; Lima: Ediciones Taraxacum, Centro Cultural Pío Aza, WALSH Ecuador.

ARELLANO, A. J., 2011 - El manejo prehispánico del paisaje en zonas marginales de la Amazonía: La Amazonía Norte del Ecuador y la zona Norte de las tierras bajas de Bolivia. 
In: Por donde hay soplo, Estudios amazónicos en los países Andinos (J.-P.Chaumeil, O. Espinoza \& M. Cornejo, eds.): 89-135; Lima: Instituto Francés de Estudios Andinos (IFEA), Pontificia Universidad Católica del Perú, CAAAP, Centre EREA du LESC.

ARELLANO, A. J. \& TAMAYO, F., 2004 - Rescate y Monitoreo de la Línea de Flujo desde Yanaquincha Oeste hasta el CPF; Quito: Informe al Instituto Nacional de Patrimonio Cultural.

BOLIAN, C. E., 1972 - An Archaeological Survey of the Trapecio of Amazonas, Colombia. Paper presented at the Northeastern Anthropological Meetings, April 21-23; Buffalo, New York.

CABODEVILLA, M. A., 1998 - Culturas de Ayer y Hoy en el Río Napo, 75 pp.; Ecuador: Cicame.

CARRERA, J., 2003 - Hacia el Suroeste de Nueva Loja. In: Cuyabeno Ancestral (M. Aguilera, J. Arellano, J. Carrera \& R. Jones, eds.): 165-203; Quito: Ediciones Simbioe, GAIA, WALSH Ecuador.

CIFUENTES, A., 2006 - Arqueología del Piedemonte Amazónico Putumayo (Colombia). In: Pueblos y Paisajes Antiguos de la Selva Amazónica (G. Morcote-Ríos, S. Mora C. \& C. Franky C., eds.): 113-130; Bogotá: Universidad Nacional de Colombia, Taraxacum.

CIFUENTES, A., 2007a - Plan de manejo Ambiental. Monitoreo Arqueológico Conga 1, Orito; Bogotá: Informe a Petrominerales Colombia Ltd.

CIFUENTES, A., 2007b - Prospección, Monitoreo y Recuperación Arqueológica DEV-7 CPI. Orito, Putumayo; Bogotá: Informe a Petrominerales Colombia Ltd.

COLLEONI, P. \& PROAÑO, J., 2010 - Caminantes de la Selva, Los pueblos en aislamiento en la Amazonía Ecuatoriana, 48 pp.; Quito: CONAIE, IPES, IWGIA. Informe IWGIA 7.

DELGADO, F. \& ACUÑA, F., 2003 - Informe del proceso de estabilización y restauración de una vasija encontrada en el monitoreo de la Plataforma Nenke, Bloque 31; Quito: Perez Companc Ecuador Cia. Ltda.

DELGADO, F., 2002 - Proyecto de Prospección Arqueológica del Pozo Nenke, Bloque 31; Quito: Informe al Instituto Nacional de Patrimonio Cultural.

DE SAULIEU, G., 2006 - Revisión del material cerámico de la colección Pastaza (Amazonía Ecuatoriana). Journal de la Société des américanistes, 92 (1-2): 279-301.

DOMINGUEZ, V., 2001a - Prospección Arqueológica de la Vía de acceso y Plataforma del Pozo Pindo 12; Quito: Informe al Instituto Nacional de Patrimonio Cutural. Petróleos Sudaméricanos. Petroriva.

DOMINGUEZ, V., 2001b - Rescate y Monitoreo Arqueológico de la Vía de acceso y Plataforma del Pozo Pindo 11; Quito: Informe al Instituto Nacional de Patrimonio Cultural. Petróleos Sudaméricanos. Petroriva, Esingeco.

ECHEVERRÍA, J., 1999 - Informe del proyecto de Investigación Arqueológica realizada en la plataforma Pata-1, en el derecho de vía de la carretera y en la plataforma Palo Azul del Bloque 18 de Cayman International Company, Región Amazónica Ecuatoriana; Quito: Instituto Nacional de Patrimonio Cultural.

ECHEVERRÍA, J., 2001 - Informe final del reconocimiento arqueológico en la ampliación de la Plataforma Ginta 1 (Ginta A) de Repsol, TPF Ecuador Inc. Bloque 16 Development, región Amazónica Ecuatoriana; Quito: Instituto Nacional de Patrimonio Cultural.

ECHEVERRÍA, J., 2004 - Exploración arqueológica en la plataforma Pata-1, comunidad de Huarataco, San Sebastián del Coca. In: Actas de los Seminarios y Talleres científicos del 30 aniversario de Orstom/IRD en Ecuador: 66-69.

EVANS, C. \& MEGGERS, B. J., 1968 - Archaeological Investigations on the Rio Napo, Eastern Ecuador, 221 pp.; Washington D.C.: Smithsonian Institution Press. 
GUAMAN, J. \& NETHERLY, P., 1996 - Análisis de la cerámica procedente del sitio NOOP30, Area Tivacuno de Maxus-Ecuador, Parque Yasuni; Quito: Informe Fundación Alexander Von Humboldt.

GUFFROY, J., 2006 - El Horizonte corrugado: correlaciones estilísticas y culturales. Bulletin de l'Institut Français d'Études Andines, 35 (3): 347-359.

MEGGERS, B. J. \& EVANS, C., 1958 - Archaeological evidence of a prehistoric migration from the Río Napo to the mouth of the Amazon. Migrations in the New World Culture History: Social Science Bulletin, 27 (21-2): 9-19.

MEGGERS, B. J., 1992 - Prehistoric Population Density in the Amazon Basin. In: Disease and Demography in the Americas. (J. W. Verano \& D. H. Ubelaker, eds.): 197-205; Washington D.C.: Smithsonian Institution Press.

MEGGERS, B. J., 1999 - Ecología y biogeografía de la Amazonía, 336 pp.; Quito: Ediciones Abya-Yala.

MORCOTE-RIOS, G., 2006 - Plantas y gentes antíguas en un igapó estacional del interfluvio Solimôes-iça (Amazonas-Putumayo). In: Pueblos y Paisajes Antiguos de la Selva Amazónica (G. Morcote-Ríos, S. Mora C. \& C. Franky C. eds.): 253-259; Washington D.C.: Universidad Nacional de Colombia, Taraxacum.

MORCOTE-RIOS, G., 2008 - Antiguos Habitantes en Ríos de Aguas Negras. Ecosistemas y cultivos en el interfluvio Amazonas-Putumayo, Colombia-Brasil, 242 pp.; Bogotá: Instituto de Ciencias Naturales, Facultad de Ciencias, Universidad Nacional de Colombia.

MORCOTE-RÍOS, G. \& SICARD, T. L., 2012 - Las Terras Pretas del Igarapé Takana. Un sistema de cultivo precolombino en Leticia-Amazonas, Colombia, 185 pp.; Bogotá: Instituto de Ciencias Naturales, Instituto de Estudios Ambientales, Universidad Nacional de Colombia.

NETHERLY, P., 1997 - Loma y ribera, Patrones de asentamiento prehistóricos en la Amazonía Ecuatoriana. Fronteras de Investigacion, 1: 33-54; Quito: Fundación Alexander Von Humboldt.

OCHOA, M., 2000 - Informe del reconocimiento arqueológico realizado en Apaika para la sísmica 3D, Bloque 31 en la provincia Francisco de Orellana a cargo de la Compañía Perez Companc S.A.; Quito: Instituto Nacional de Patrimonio Cultural.

OCHOA, M., 2003 - Informe del rescate y monitoreo arqueológico del sitio Eden, Bloque 15, Provincia Orellana; Quito: Instituto Nacional de Patrimonio Cultural.

OCHOA, M., 2007 - Redefiniendo la Fase Pastaza. In: II Congreso Ecuatoriano de Antropología y Arqueología, Balance de la última década: Aportes, Retos y nuevos temas, 1: 463-492; Quito: Ediciones ABYA-YALA, Banco Mundial.

ORTÍZ DE VILLALBA, J. S., 1981 - Antiguas Culturas Amazónicas, Fase Napo (1188-1480 d.C.), 70 pp.; Ecuador: Cicame.

PORRAS, P., 1975 - El formativo en el valle amazónico del Ecuador. La fase Pastaza. Revista de la Universidad Católica de Quito, 3 (10): 74-134.

PORRAS, P., 1982 - Arqueología de Quito, Fase Cotocollao, 267 pp.; Quito: Artes Gráficas Señal.

PORRAS, P., 1987 - Investigaciones Arqueológicas a las faldas del Sangay, 432 pp.; Quito: Artes Gráficas Señal.

RENARD-CASEVITZ, F.-M., SAIGNES, T. \& TAYLOR, A.C., 1988 - Al Este de los Andes, Tomo II, 265 pp.; Quito: Ediciones Abya Yala, Institut Français d'Études Andines (IFEA). 
ROSTAIN, S., 1999 - Excavación en área en un montículo de Huapula, Amazonía Ecuatoriana (Proyecto Sangay-Upano). In: Memorias del Primer Congreso Ecuatoriano de Antropología, Vol. III (E. Salazar, ed.): 227-256; Quito: Museo Jacinto Jijón y Caamaño.

ROSTAIN, S., 2006 - Etnoarqueología de las casas Huapula y Jívaro. Bulletin de I'Institut Français d'Études Andines, 35 (3): 337-346.

ROSTAIN, S., 2010 - Cronología del valle del Upano (Alta Amazonía ecuatoriana). Bulletin de l'Institut Français d'Études Andines, 39 (3): 667-681.

ROSTOKER, A., 2003 - Formative Period Chronology for Eastern Ecuador. In: Archaeology of Formative Ecuador (J. Quilter, ed.): 539-545; Washington D.C.: Dumbarton Oaks Research Library and Collection.

SALAZAR, E., 1999 - De vuelta al Sangay, Investigaciones arqueológicas en el Alto Upano. In: Memorias del Primer Congreso Ecuatoriano de Antropología, Vol. III (E. Salazar, ed.): 184-225; Quito: Museo Jacinto Jijón y Caamaño.

SALAZAR, E. \& OCHOA, M., 2003 - Informe de la Prospección y Rescate y Monitoreo Arqueológico del Campo Eden-Yuturi. Bloque 15, Provincia Francisco de Orellana; Quito: Instituto Nacional de Patrimonio Cultural.

SALAZAR, E., ARELLANO, A. J. \& OCHOA, M., 2000 - Reconocimiento Arqueológico del Sector Limoncocha y Zonas Adyacentes, Provincias de Orellana y Sucumbíos; Quito: Informe al Instituto Nacional de Patrimonio Cultural.

SALAZAR, E., ARELLANO, A. J., OCHOA, M. \& MANOSALVAS, O., 1999 - Informe del Reconocimiento Arqueológico de la Línea del Oleoducto Ecuatoriano y Zonas Adyacentes; Quito: Instituto Nacional de Patrimonio Cultural.

SÁNCHEZ, A., 2002 - Monitoreo del componente arqueológico durante la construcción de las vías de acceso y plataformas de los pozos Yuca Sur 12 y Yuca Sur 13 (Ex Florida 01), en el camino Palanda. Provincia de Orellana; Quito: Novoambiente.

SOLÓRZANO, V., M. S., 2006 - Análisis de la distribución de los asentamientos registrados mediante arqueología de contrato en la Provincia Orellana, Ecuador. Arqueología y Territorio, 3: 39-57.

SOLÓRZANO, V., M.S., 2007 - Arqueología de Contrato, una forma de minimizar el impacto durante el proceso de remoción de suelo. Estudio de caso en Orellana (Ecuador). Praxis Archaeologica, 2: 107-128.

URIBE, M. V., 1980-1981 - Reconocimiento arqueológico del valle medio del río Guamués (Putumayo). Revista Colombiana de Antropología, 23: 253-273.

VILLALBA, M., 1988 - Cotocollao, 571 pp.; Quito: MAE Serie Monográfica 2.

VILLALBA, M., 2000 - Informe de la Investigación Arqueológica en las plataformas para los pozos de Desarrollo Nantu 4, Sunka 3 y Penke 3 del Bloque 14, Operado por Vintage Oil Ecuador S. A.; Quito: Informe al Instituto Nacional de Patrimonio Cultural.

VILLALBA, M., 2003 - Reconocimiento y Prospección Arqueológica en la plataforma del Pozo Auca 53 y Vía de Acceso, Provincia de Orellana; Quito: Informe al Instituto Nacional de Patrimonio Cultural.

ZÁRATE, C. G., 1998 - Movilidad y permanencia Ticuna en la frontera amazónica colonial del siglo XVIII. Journal de la Société des Américanistes, 84 (1): 73-98. 